\title{
Structural Equivalence of Context-Free Grammar Forms is Decidable
}

HERMANN $K_{1}-G$. WALTER

TECHNISCHE HOCHSCHULE DARMSTADT

WEST GERMANY

\section{ABSTRACT:}

Two grammars $G_{1}$ and $G_{2}$ are structural equivalent if the corresponding parenthesized grammars generate the same language. This definition transfers to grammar forms in a natural way. It is shown that structural equivalence of context-free grammar forms is decidable.

\section{KEY WORDS AND PHRASEs:}

Contextfree grammars, parenthesized grammars, grammar forms, grammar homomorphisms, reduction, grammatical language families.

CR Categories: $4.12,5.23,5.25$ 


\section{O. INTRODUCTION,}

In [2] Cremers and Ginsburg define grammar families by the concept of grammar forms. Intuitively a grammar form F defines a gramar family such that any member of this family is "similar" to a fixed prototype grammar $G_{F}$. Two natural equivalence problems arise by these definitions, first the problem wether two grammars $G_{F}$ and $G_{F}$ generate the same grammar family (strong equivalence) and second the problem wether two grammars $G_{F_{1}}$ and $G_{F_{2}}$ generate the same language family (weak equivalence).

In [2] it is shown that the first problem is decidable. The second one is still open. We want to show that another equivalence problem which is more general than strong equivalence and less general than weak equivalence is decidable.

Following [5] we introduce the structural equivalence of two grammars $G_{1}$ and $G_{2}$. $G_{1}$ and $G_{2}$ are structural equivalent if the associated parenthesized grammars generate the same language. This definition transfers in a natural way to grammax forms. It is known $([5],[6])$ that structural equivalence of context-free grammars is decidable. we generalize this result to structural equivalence of context-free grammar forms.

\section{BASIC NOTATIONS,}

We adopt the usual notations of phrase structure gramars $G=(V, \Sigma, P, \sigma)$, context-free grammars, etc. ([3], [6]) with the (trivial) change $\sigma: V-\Sigma$. To avoid trivialities we assume (without loss of generality), that all grammars in consideration are reduced $([3],[6]$ ) and contain no unnecessary symbols.

By $+(\longmapsto)$ we denote the notion of a (direct) derivation. The set $\mathcal{L}(G)=\left\{w \in \Sigma^{*} \mid \exists \sigma_{1} \in \sigma: 0 \ldots w\right\}$ is the generated language and

$$
D(G)=\left\{\sigma_{1}+w \mid w \in \Sigma^{*} \quad \& \sigma_{1} \in 0\right\}
$$

is the set of derivations. 
A grammar morphism $\phi: G_{1} \rightarrow G_{2}$ is a monoid homomorphism

$\phi: v_{1}^{*} \rightarrow v_{2}^{*}$ with

$$
\left(\sigma_{1}\right) \subseteq \sigma_{2}
$$

$$
\phi\left(v_{1}-\Sigma_{1}\right) E\left(v_{2}-\Sigma_{2}\right)
$$

$$
\phi\left(\Sigma_{1}\right) E \Sigma_{2}^{*}
$$

We single out various classes of gramar morphisms.

If $\phi$ is lengthpreserving, we call $\phi$ a fine morphism, if

$\phi(\xi)=\xi\left(\xi \in v_{1}-\Sigma_{1}\right) \phi$ is external and if $\phi(t)=t\left(t \in \Sigma_{1}\right) \phi$ is internal.

In a natural way $\phi$ induces $\hat{\phi}: D\left(G_{1}\right) \rightarrow D\left(G_{2}\right) \cdot([4])$

We call $\phi$ closed if $\hat{\phi}$ is surjective. For closed morphisms $\phi$ we get: $\phi\left(\chi\left(G_{1}\right)\right)=\mathcal{L}\left(G_{2}\right)$. A closed, internal morphism $\phi$ is called a reduction. $\phi$ is an isomorphism if $\phi$ and $\phi^{-1}$ are morphisms.

To any grammar $G$ we associate a grammar family ${ }^{*} G$ to be the collection of all grammaxs $\mathrm{G}^{\prime}$ such that there exists a diagram

$$
G<\frac{\phi_{1}}{G^{\prime \prime}} \stackrel{ }{\phi_{2}} \mathrm{G}^{\prime}
$$

where $\phi_{1}$ is fine and $\phi_{2}$ is external and closed. By $\lambda(G)$ we denote the collection of all languages $\mathcal{L}\left(G^{\prime}\right)$ with $G^{\prime} \in \Gamma_{G}$

The existence of such a diagram is by [10] equivalent to the notion of grammar forms [2] and therefore $\lambda(G)$ is exactly the grammatical language family ([2]) associated to this grammar form. We call $G_{1}$ and $G_{2}$ strong equivalent $\left(G_{1} \approx G_{2}\right)$ if $\Gamma_{G_{1}}=\Gamma_{G_{2}}$, and weak equivalent $\left(G_{1} \sim G_{2}\right)$ if $\lambda\left(G_{1}\right)=\lambda\left(G_{2}\right)$.

Now, consider a universal bracket pair $\{()$,$\} .$

If $G$ is a grammar, then the associated parenthesized gramar $G$ () is obtained by

$$
G^{()}=(\nabla \cup\{(,)\}, \Sigma \cup\{(,)\},\{(p,(q)) \mid(p, q) \in p\}, \sigma)([5],[6]) .
$$

Denote by $\lambda^{()}(G)$ the collection of all languages $\mathcal{L}\left(G^{\prime}\right)$, with $G^{\prime} \in \Gamma_{G^{*}}$

$G_{1}$ and $G_{2}$ are called structural equivalent $\left(G_{1} \equiv G_{2}\right)$ if $\lambda^{()}\left(G_{1}\right)=\lambda^{()}\left(G_{2}\right)$. 
It is easy to check:

$$
G_{1} \approx G_{2} \Longrightarrow G_{1} \equiv G_{2} \Longrightarrow G_{1} \sim G_{2} \text {. }
$$

Example: Consider $G_{1}$ and $G_{2}$ specified by

$$
\begin{aligned}
\mathrm{P}_{1}=\sigma & \rightarrow \sigma_{1}+\sigma_{1} \\
\mathrm{P}_{2}=\sigma & +\sigma_{1} \sigma_{1}+\sigma_{1}+\varepsilon \\
& +\sigma_{1}+\varepsilon
\end{aligned}
$$

It is easy to check that $\lambda\left(G_{1}\right)=\mathcal{L}_{\text {reg }}=\lambda\left(G_{2}\right), \lambda^{()}\left(G_{2}\right) \sum_{\text {in }}$ and $D_{1} \in \lambda^{()}\left(G_{1}\right)$, where $D_{1}$ is the Dyck Ianguage over $\{()$,$\} , hence$ $G_{1} \sim G_{2}$ but $G_{1} \neq G_{2}$.

From now on, we assume without further mentioning all grammars to be context-free.

\section{PRELIMINARY RESULTS,}

In this section we derive some results on grammar morphisms and parenthesized grammars, which are necessary to prove our main result. we state without proof.

Iemma 1: Let $\phi: G_{1} \rightarrow G_{2}$ be a morphism There exists a factorization $\phi=\phi_{1} \circ \phi_{2}$ resp. $\phi_{2} \circ \phi_{1}$ with $\phi_{2}$ internal and $\phi_{1}$ external. If $\phi$ is closed then $\phi_{2}$ is closed.

Eemma 2: If $\phi_{1}: G_{0} \rightarrow G_{1}$ is closed and external and $\phi_{2}: G_{0} \rightarrow G_{2}$ is internal, then there exist $\psi_{1}: G_{2} \rightarrow G_{3}$ closed and external and $\psi_{2}: G_{1} \rightarrow G_{3}$ internal with $\psi_{2} \phi_{1}=\psi_{1} \phi_{2}$. Moreover, if $\phi_{1}$ is fine then $\psi_{1}$ is fine and if $\phi_{2}$ is a reduction then $\psi_{2}$ is a reduction.

We now study the effect of parenthesizing. Obviously there is a canonical external and closed morphism $e: G() \rightarrow G$ defined via bracket erasing.

Consider a morphism $\phi: G_{1} \rightarrow G_{2}$, then $\phi$ induces a unique morphism $\phi^{()}=G_{1}^{()} \rightarrow G_{2}^{()}$such that

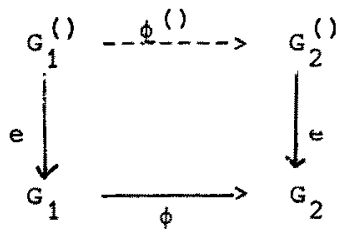


is commutative. Observe that in a certain sense parenthesizing is a functor. Obviously properties of $\phi$ transfer to $\phi()$, especially if $\phi$ is a reduction then $\phi^{()}$is a reduction.

This yields

Fact: If $\phi: G_{1} \rightarrow G_{2}$ is a reduction, then $\mathcal{X}\left(G_{1}^{()}\right)=\mathcal{X}\left(G_{2}^{\prime}\right)$.

Conversely if $\phi: G_{1}^{()} \rightarrow G_{2}^{()}$is a "bracket" morphism $(\phi(()=(\& \phi())=))$, then $\phi$ induces $\hat{\phi}: G_{1} \rightarrow G_{2}$ with $\phi=\hat{\phi}()$. Again properties of $\phi$ transfer to $\hat{\phi}$.

call a grammar $G$ invertible $i f(p, q) \&\left(p, q^{\prime}\right) \in p \Longrightarrow q^{\prime}=q^{\prime}$.

Lemma 3: To any grammar G there exists a diagram

$$
G \ll \stackrel{\phi_{1}}{\longrightarrow} G_{0} \stackrel{\phi_{2}}{\longrightarrow} G^{i n v}
$$

where $\phi_{1}$ and $\phi_{2}$ are reductionsand $G^{i n v}$ is invertible.

Proof: We analyze the construction of $\mathrm{G}^{i n v}$ given in [5]. [6].

Step 1: "Ginv":

consider $z^{*}=2^{V-\Sigma}-\{\not\}$. Define a substitution $u=\left(z^{\prime} v \Sigma\right)^{*} \rightarrow 2^{V^{*}}$ by

$$
u\left(z_{1}\right)=\left\{\begin{array}{c}
z_{1}, z_{1} \in z^{\prime} \\
\left\{z_{1}\right\}, z_{1} \in \Sigma .
\end{array}\right.
$$

to any $q \in\left(z^{*} \cup \Sigma\right)^{*}$ consider

$$
s_{1}=\left\{\xi \mid q^{\prime} \text { with } \xi \rightarrow q^{\prime} \in \text { p \& } q^{\prime} \in \mu(q)\right\} \text {. }
$$

Now define $\mathrm{G}^{\text {inv }}$ as follows

$$
\mathrm{G}^{\mathrm{inv}}=\left(\mathrm{V}^{\prime}, \Sigma, \mathrm{P}^{\prime}, \sigma^{*}\right)
$$

$$
\begin{aligned}
& v^{\prime}-\Sigma=\left\langle s_{q}\right| s_{q} \neq \phi, q=t_{0} s_{q_{1}} t_{1} \ldots, s_{q_{i}} t_{i} \\
& \text { with } \left.\mathrm{s}_{q_{\lambda}} \neq \phi, t_{\lambda} \in \Sigma^{*}, q_{,} q_{\lambda} \in\left(z^{\prime} \cup \Sigma\right)^{*}, 0 \leqslant \lambda \leqslant i\right\}
\end{aligned}
$$

(ii) $p^{\prime}$

$=\left(\left(s_{q}, q\right) \mid q \in\left(z^{\prime} \cup \Sigma\right)^{*} \& s_{q} \in v^{\prime}-\Sigma\right)$

(iii) $\sigma^{\prime}$

$$
=\left\{s_{q} \mid s_{q} \cap \sigma \neq \varnothing\right\}
$$

It is easy to check, that $\mathrm{G}^{i n v}$ is invertible. 
Step 2: "Go" $\phi_{1}, \phi_{2} "$

Define $G_{0}=\left(V_{0}, \Sigma, P_{0}, \sigma_{0}\right)$ by

(i) $\quad v_{0}-\Sigma=\left\{\left(s_{q}, \xi\right) \mid \xi \in s_{q}, q \in v^{*}-\Sigma\right\}$

(ii) $\sigma_{0} \quad=\left\{\left(s_{q}, \sigma_{1}\right) \mid \sigma_{1} \in \sigma \cap s_{q}\right\}$

(iii) $\mathrm{P}_{0} \quad=\left\{\left(s_{q^{\prime}}, \xi\right) \rightarrow t_{0}\left(s_{q_{1}}, n_{1}\right), \ldots,\left(s_{q_{i}}, n_{i}\right) t_{i}\right\}$

a) $i \geqslant 0, t_{\lambda} \in \Sigma^{*}(0 \leqslant \lambda \leqslant i)$

B) $\xi \in s_{q^{\prime}}, \eta_{\lambda} \in s_{q_{\lambda}}(1 \leqslant \lambda \leqslant i)$

r) $q=t_{0} s_{q_{1}}, \ldots, s_{q_{i}} t_{i}$

d) $\left.\xi \rightarrow t_{0} n_{1} \ldots n_{i} t_{i} \in P\right\}$.

Induce $\phi_{1}$ and $\phi_{2}$ by $\phi_{1}\left(\left(s_{q}, \xi\right)\right)=\xi$ and $\phi_{2}\left(\left(s_{q}, \xi\right)=s_{q}\right.$.

Step 3: " $\phi_{1}$ is a reduction"

We observe first for all $\sigma_{1} \in \sigma$

$$
\phi_{1}^{-1}\left(\sigma_{1}\right)=\left\{\left(s_{q}, \sigma_{1}\right) \mid \sigma_{1} \in s_{q}\right\} \quad \underline{r} \sigma_{0} .
$$

Now consider $\left(s_{q}, \xi\right) \rightarrow t_{0}\left(s_{q}, \eta_{1}\right) \ldots\left(s_{q_{i}}, n_{i}\right) t_{i} \in P_{0}$ and

$q^{\prime}=t_{o}^{\prime}\left(s_{q_{1}^{\prime}}, n_{i}^{\prime}\right) \ldots\left(s_{q_{j}^{\prime}}, n_{j}^{\prime}\right) t_{j}$ such that

$$
t_{0} n_{1} \cdots n_{i} t_{i}=\phi_{1}\left(q^{\prime}\right)=t_{j} n_{i}^{\prime} \cdots n_{j}^{\prime} t_{j}^{\prime}
$$

we conclude $j=i, t_{0}=t_{0}^{\prime}, \ldots, t_{i}=t_{i}^{\prime}, n_{1}=n_{i}, \ldots, n_{i}=n_{i}$.

On the other hand, by $\xi \rightarrow t_{0} n_{1} \cdots n_{i} t_{i} \in P$ we get $\xi \in s_{q}$, hence

$$
\left(s_{q^{\prime}}, \xi\right) \rightarrow t_{o}\left(s_{q_{1}}, n_{1}\right) \ldots\left(s_{q_{i}}, \eta_{i}\right) t_{i} \in p_{0} \text {. }
$$

This proves, that $\phi_{1}$ is coperfect in the sense of $[11]$. Hence $\phi_{1}$ is a reduction ([11]).

Step 4: "\$2 is a reduction"

Again, we observe the following fact.

If $s_{q} \in \sigma^{\prime}$, then there exists $\sigma_{1} \in \sigma \curvearrowright s_{q}$, hence $\left(s_{q}, \sigma_{1}\right) \in \sigma_{0}$.

This proves $\phi_{2}\left(\sigma_{0}\right)=\sigma^{\prime}$. 


\section{Consider}

and

$$
\left(s_{q}, \xi\right) \rightarrow t_{0}\left(s_{q_{i}}, n_{1}\right) \ldots\left(s_{q_{i}}, n_{i}\right) t_{i} p_{o}
$$

$\left(s_{q}, \xi^{\prime}\right)$ with $\phi_{2}\left(s_{q^{\prime}}, \xi^{\prime}\right)=\phi_{2}\left(\left(s_{q}, \xi\right)\right)$.

. We get $s_{q}=s_{q^{\prime}}$. Since $\xi^{\prime} \epsilon s_{q}$ and $q=t_{0} s_{q_{1}} \ldots s_{q_{i}} t_{i}$ there exist $\eta_{1} \ldots \eta_{i}^{\prime}$ with $\xi^{\prime} \rightarrow t_{0} \eta_{1}^{\prime} \ldots \eta_{i}^{i} t_{i} \in$ and $\eta_{\lambda}^{\prime} \in s_{q_{\lambda}}(1 \leqslant \lambda \leqslant i)$.

We conclude

$$
\left(s_{q}, \xi^{\prime}\right) \rightarrow t_{0}\left(s_{q_{1}}, n_{i}^{\prime}\right) \ldots\left(s_{q_{i}}, n_{i}^{\prime}\right) t_{i} \in p_{o}
$$

and

$$
\phi_{2}\left(t_{\rho}\left(s_{q_{1}}, n_{1}^{\prime}\right) \ldots\left(s_{q_{i}}, n_{i}^{\prime}\right) t_{i}\right)=\phi_{2}\left(t_{o}\left(s_{q_{1}}, \eta_{1}\right) \ldots\left(s_{q_{i}}, \eta_{i}\right) t_{i}\right) \cdot
$$

This proves that $\phi_{2}$ is perfect in the sense of $[11]$. Hence $\phi_{2}$ is a reduction ([1 1$]$ ).

We are now able to prove one of the key theorems.

Theorem 1: The following statements are equivalent for two grammars $G_{1}$ and $G_{2}$

(1) $\quad y\left(G_{1}^{()}\right)=\mathcal{L}\left(G_{2}^{()}\right)$

(2) There exists a diagram

$$
\mathrm{G}_{1} \ll \phi_{1} \mathrm{G}_{\mathrm{O}} \underset{\phi_{2}}{\longrightarrow} \mathrm{G}_{2}
$$

where $\phi_{1}$ and $\phi_{2}$ are reductions.

Proof: $(2)=(1)$ with the above fact.

(1) $\Longrightarrow$ (2). BY lemma 3 there exist two diagrams

$$
G_{i}<\frac{G_{i}^{i}}{G_{i}} \longrightarrow G_{i}^{i n v} \quad(i=1,2)
$$

with $\psi_{1}^{i}$ and $\psi_{2}^{i}$ reductions $(i=1,2)$.

Parenthezising leads to diagrams

$$
G_{i}^{()}<\frac{\hat{\psi}_{i}^{i}}{G_{i}^{(1)}} \underset{\hat{\psi}_{2}^{i}}{\left(G_{i}^{n}()\right.} \quad(i=1,2)
$$

where $\psi_{1}^{i}$ and $\psi_{2}^{i}$ are reductions and $G_{i}^{*}()$ is invertible $(i=1,2)$. since

$$
\mathcal{L}\left(G_{1}^{n}()\right)=\mathcal{L}\left(G_{1}^{0}\right)=\mathcal{L}\left(G_{2}^{()}\right)=\mathcal{L}\left(G_{2}^{\prime \prime}\right)
$$

we obtain from [5], that there exists an internal isomorphism between 
$G_{1}^{\prime \prime)}$ and $G_{2}^{\prime \prime}$.

Hence we get a diagram

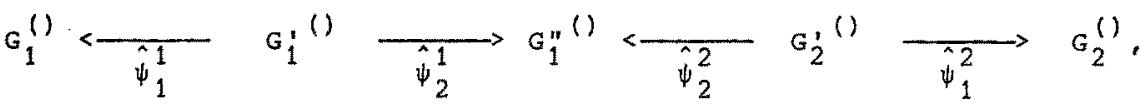

where all arrows are reductions.

Removing the brackets, we get a diagram

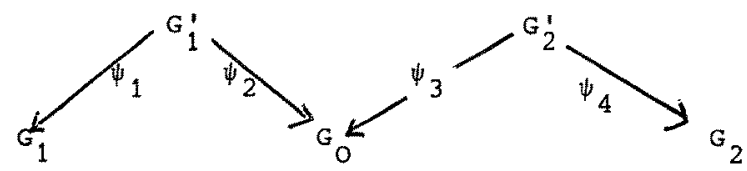

where all $\psi_{i}(i=1,2,3,4)$ are reductions.

By a theorem of [8]. [9] we can fill in this aiagram with reductions $\psi_{5}$ and $\psi_{6}$.

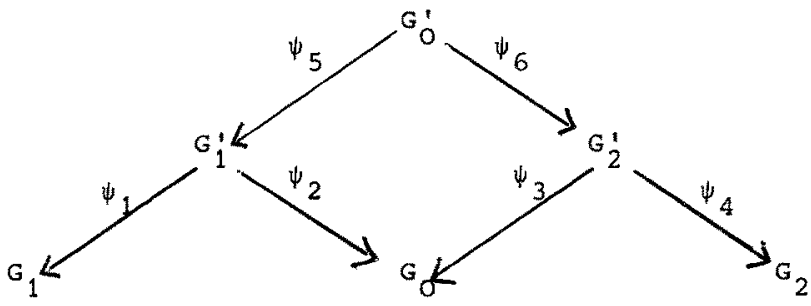

Now, letting $\phi_{1}=\psi_{1} \psi_{5}$ and $\phi_{2}=\psi_{4} \psi_{6}$, we get the theorem.

\section{THE MAIN THEOREM}

We are now in the position to prove a characterization of the structural equivalence.

Theorem 2: The following statements are equivalent for two grammaxs $G_{1}$ and $G_{2}$ :
(1) $\lambda^{()} G_{2} \subseteq \lambda^{()}\left(G_{1}\right)$
(2) There exists a diagram

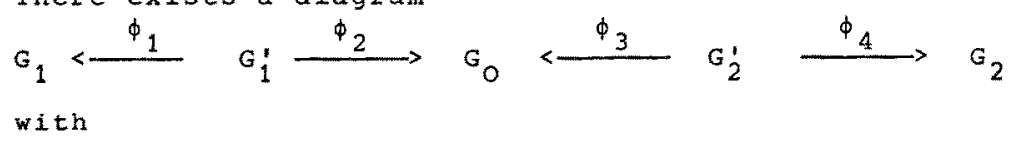


(a) $\phi_{1}$ is internal

(B) $\phi_{2}$ is a reduction

( $\gamma$ ) $\phi_{3}$ is fine, external and closed

(b) $\phi_{4}$ is external and closed.

Proof: $(1) \Rightarrow$ (2)

Consider $G_{2}$. Since $\mathcal{L}\left(G_{2}^{()}\right) \in \lambda^{()}\left(G_{1}\right)$, there is a $\hat{G}_{2} \in \Gamma_{G_{1}}$ with $\mathcal{L}\left(\hat{G}_{2}^{()}\right)=\mathcal{L}\left(G_{2}^{()}\right)$.

By theorem 1 and the definition of $P_{G_{1}}$ we get a diagram

$G_{1} \longleftarrow$ fine $\hat{G}_{1} \frac{\text { closed }}{\text { external }}>\hat{G}_{2}<\frac{}{\text { reduction }} \hat{\hat{G}}_{2} \stackrel{ }{\text { reduction }} G_{2}$

consider the diagram

$\hat{G}_{1} \frac{\text { closed }}{\text { external }} \hat{G}_{2} \underset{\text { reduction }}{\longrightarrow} \hat{\mathrm{G}}_{2}$.

By a theorem of [10] we can fill in this diagram in the following way

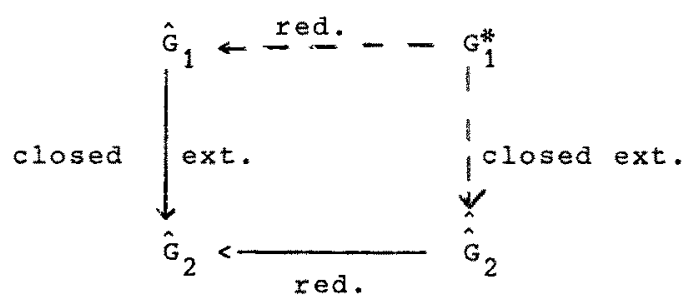

Consider the diagram

$G_{1}^{*} \stackrel{\text { closed }}{\text { ext. }} \rightarrow \hat{\hat{G}}_{2} \stackrel{ }{\text { red. }} G_{2}$.

By lemma 1 we can fill in this diagram in the following way

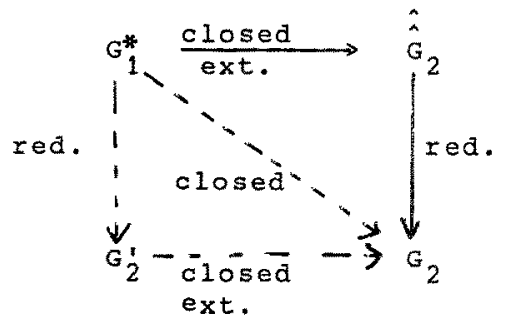


Now, we reached the following situation:

$$
G_{1} \ll \frac{\text { fine }}{\text { red. }} G_{1}^{*} \quad G_{2}^{\prime} \quad \frac{\text { ext. }}{\text { closed }} G_{2}
$$

By lemma 1 again we find a diagram

$$
G_{1}<\frac{\text { internal }}{G_{1}} \underset{<i n e}{<\text { ext. }} G_{1}^{*} \text {. }
$$

Consider the diagram

$$
G_{1}<\frac{\text { ext. }}{\text { fine }} G_{1}^{*} \underset{\text { red. }}{\longrightarrow} G_{2}^{\prime} \text {. }
$$

By lemma 2 we can fill in this diagram:

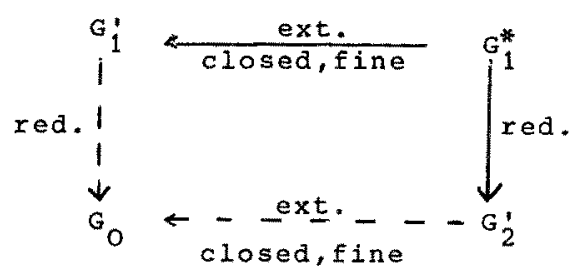

But this proves the if-part of theorem 2 .

(2) $\Longrightarrow$ (1) Consider an arbitrary diagram

$$
G_{2}<\text { fine } G_{3} \frac{\text { ext. }}{\text { closed }} G_{3}
$$

By a theorem of [10] we get a diagram

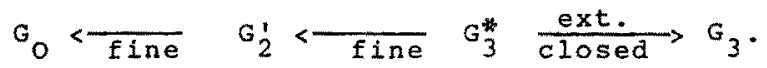

on the other hand by a theorem of [8], [9] we get a diagram

$$
G_{1}<\frac{\text { fine }}{G_{3}^{*}} \underset{\text { red. }}{\longrightarrow} G_{3}^{*} \text {. }
$$

In summary we obtain

$$
\mathrm{G}_{1} \ll \frac{}{\text { fine }} \mathrm{G}_{3}^{*} \underset{\text { red. }}{\longrightarrow} \mathrm{G}_{3}^{*} \frac{\text { ext. }}{\text { closed }}>\mathrm{G}_{3}
$$

By definition: $G_{3}^{* *} \in \Gamma_{G_{1}}$. By section 2 we get

$$
\mathcal{L}\left(\mathrm{G}_{3}^{*}()\right)=\mathcal{L}\left(\mathrm{G}_{3}^{*}()\right) \text { and } \mathcal{L}\left(\mathrm{G}_{3}^{()}\right)=\mathrm{h}\left(\mathcal{L}^{*}\left(\mathrm{G}_{3}^{*}\right)\right)
$$

where $h$ is a certain, bracket preserving homomorphism. But this proves $\left.\mathcal{L}_{\left(\mathrm{G}_{3}()\right.}\right) \in \lambda^{()}\left(\mathrm{G}_{1}\right)$. 
It remains to show the decidability of statement (2) of theorem 2 . Denote by $\| G||=\max |q|$. To any $G$ and $z$ with $z \cap \Sigma=\varnothing$ $(p, q) \in p$

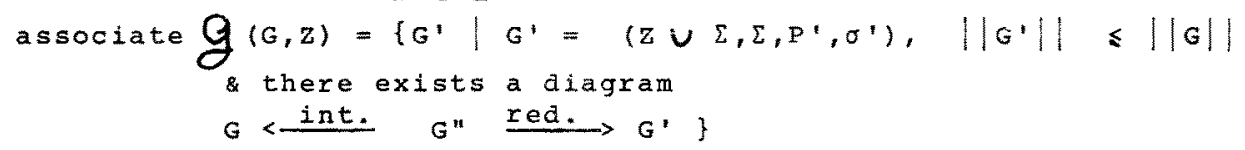

Lemma 4: $|g(G, z)|<\infty$ and there is an algorithm which computes $g(G, z)$.

Proof: We show first, that to any diagram

$$
G<\frac{\text { int. }}{\phi_{1}} G^{\prime \prime} \frac{\text { red. }}{\phi_{2}} G^{\prime}
$$

exists a diagram

$G<$ int. $G^{\prime \prime} \stackrel{\text { red. }}{\longrightarrow} G^{*}$

with V'' $-\Sigma \subseteq(V-\Sigma) \times\left(V^{\prime}-\Sigma\right)$.

To prove this we consider the following equivalence relation on V" $-\Sigma$ :

$$
\xi \equiv \xi^{\prime} \longleftrightarrow \phi_{1}(\xi)=\phi_{1}\left(\xi^{\prime}\right) \& \phi_{2}(\xi)=\phi_{2}\left(\xi^{\prime}\right) .
$$

Then define G" $=\left\{\left\{[\xi]_{\equiv} \mid \xi \in V^{\prime \prime}-\Sigma\right\} \cup \Sigma, \Sigma, P^{\prime \prime},\left\{\left[\sigma_{1}\right]_{\equiv} \mid \sigma_{1} \in \sigma^{\prime \prime}\right\}\right)$ where

if and only if

$$
[\xi]_{\equiv} \rightarrow t_{0}\left[n_{1}\right]_{\equiv} \cdots\left[n_{i}\right]_{\equiv} t_{i} \in P * \cdot
$$

$$
\xi \rightarrow t_{0} n_{1} \cdots n_{i} t_{i} \in P^{\prime \prime}
$$

for all $\xi, n_{1}, \ldots, n_{i} \in V^{\prime \prime}-\Sigma, t_{0}, \ldots, t_{i} \in \Sigma^{*}$ and $i \geqslant 0$.

By definition of $\xi \equiv \xi^{\prime}$ we get in a natural way internal $\phi_{1}$ and $\phi_{2}$ such that

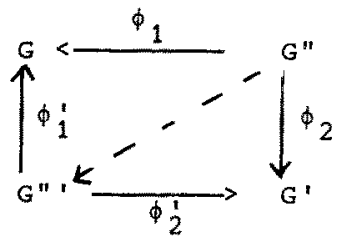

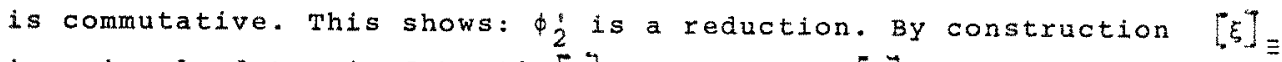
is uniquely determined by $\left.\phi_{1}^{\prime}(\xi \xi]_{\equiv}\right)$ and $\phi_{2}^{\prime}([\xi] \equiv)$. This proves the above assertion. 
To compute $g(G, Z)$ we now have to inspect only (G", G'), such that

$$
\Sigma^{\prime \prime}=\Sigma^{\prime}=\Sigma
$$

$$
V^{\prime \prime}-\Sigma 5(V-\Sigma) \times\left(V^{2}-\Sigma\right)
$$

$$
\|G\| \leqslant\|G\| \text {. }
$$

$$
|| G \cdot|| \leqslant|| G||
$$

obviously, thexe are only a finite number of such pairs and the set of all these $\left(G^{\prime \prime}, G^{\prime}\right)$ can be effectively constructed. To any such pair associate

$$
\phi_{1}: V^{\prime \prime}-\Sigma \rightarrow V-\Sigma \text { and } \phi_{2}: V^{\prime \prime}-\Sigma \rightarrow V^{\prime}-\Sigma
$$

by $\phi_{1}(\xi, n)=\xi$ and $\phi_{2}(\xi, n)=\eta \quad\left((\xi, n) \in V^{\prime \prime}-\Sigma\right)$.

We can deaide wether $\phi_{1}$ and $\phi_{2}$ define internal morphism. Now, if both are morphisms we can decide by an algorithm due to C.P. Schnorr [7] wether $\phi_{2}$ is a reduction.

Now, consider a grammar $G$ and $\Sigma^{\prime}$ with $\left(V-\sum\right) \cap \Sigma^{\prime}=\varnothing$ and $k \ll z_{+}$. To any such triple associate

$$
\begin{aligned}
X\left(G, \Sigma^{\prime}, k\right)=\left\{G^{\prime} \mid G^{\prime}\right. & =\left(\Sigma \Sigma^{\prime} \vee(V-\Sigma), \Sigma^{\prime}, P^{\prime}, \sigma\right), \\
|| G^{\prime}|| \leqslant k \text { \& there exists a diagram } & \\
G^{\prime} & \left.<\frac{\text { closed }}{\text { ext.fine }} G^{\prime \prime} \frac{\text { closed }}{\text { ext. }} \mathrm{G}\right\}
\end{aligned}
$$

Lemma 5: $|\oint(G, \Sigma,, k)|<\infty$ and there is an algorithm which computes $X\left(\mathrm{G}, \Sigma^{\prime}, k\right)$

proof: We show first, that to any diagram

$$
G^{\prime}<\frac{\text { elosed }}{\text { ext.fine }} \underset{\phi_{1}}{\frac{\text { closed }}{\phi_{2}}} \rightarrow G
$$

there exists a diagram

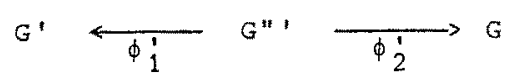

where $\phi_{i}$ is closed, external and fine and $\phi_{2}$ is closed and external, such that

$$
\Sigma^{n}=\Sigma^{\prime} \times \sum_{j=0}^{|j G| \mid} \Sigma^{j} .
$$

observe first that $\left|\phi_{2}(t)\right| \leqslant|| G||$ for all $t \in \Sigma "$. Now consider the following equivalence relation on $\Sigma "$ : 


$$
t \equiv t^{\prime} \text { iff } \phi_{1}(t)=\phi_{1}\left(t^{\prime}\right) \& \phi_{2}(t)=\phi_{2}\left(t^{\prime}\right)
$$

Define $G^{\prime \prime}=\left(\left\{\left[t_{d}\right] \mid t \in \Sigma "\right\} V\left(V^{\prime \prime}-\Sigma^{\prime \prime}\right),\left\{[t] \equiv \mid t \in \Sigma^{\prime \prime}\right\}, P^{\prime \prime}, \sigma^{\prime \prime}\right)$ where

if

$$
\xi \rightarrow n_{0}\left[t_{1}\right] \equiv \cdots\left[t_{i}\right] n_{i} \in P^{\prime \prime}
$$

$$
\xi \rightarrow n_{0} t_{1} \cdots t_{i} n_{1} \in p "
$$

for all $n_{0}, \ldots, n_{i} \in(v "-\Sigma ")^{*}, t_{1}, \ldots, t_{i} \in \Sigma n$ and $i \geqslant 0$.

Again we obtain by construction a commutative diagram

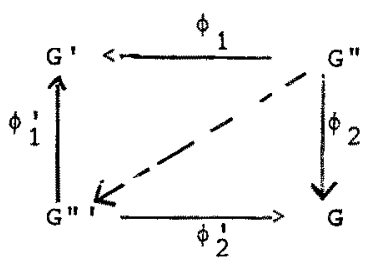

where $\phi_{1}^{\prime}$ is closed, external and fine and $\phi_{2}^{\prime}$ is closed and external. Moreover $[t]_{\equiv}$ is now uniquely determined by $\phi_{1}\left([t]_{\equiv}\right)$ and $\phi_{2}^{\prime}\left([]_{\equiv}\right)$. To compute $\mathcal{Q}\left(G^{\prime} \Sigma^{\prime}, k\right)$ we have to consider all pairs (G", G') with

(i) $\|G \cdot\| \leqslant k,\|G\| \| k$

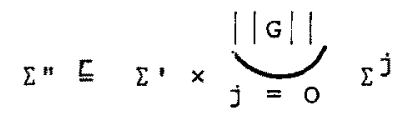

(iii) $V^{\prime \prime}-\Sigma^{\prime \prime}=V^{\prime}-\Sigma^{\prime}=V-\Sigma$.

Obviously, there are only a finite number of such pairs and all these pairs can be constructed effectively. Now, associate to any such pair (G", G') two mappings $\phi_{1}$ and $\phi_{2}$ by $\phi_{1}(t, w)=t$ and $\phi_{2}(t, w)=$ for all $(t, w) \in \Sigma^{\prime \prime}$. Decide wether both mappings define external morphisms. If they do, check: $P^{\prime}=\phi_{1}\left(P^{\prime \prime}\right)$ and $P=\phi_{2}\left(P^{\prime \prime}\right)$ which is a necessary and sufficient condition for $\phi_{1}$ resp. $\phi_{2}$ to be closed.

Consider now a diagram from theorem 2(2)

$G_{1} \stackrel{\text { int. }}{\longrightarrow} G_{1}^{\prime} \stackrel{\text { red. }}{\longrightarrow} G_{0}<\frac{\text { closed }}{\text { ext.,fine }} G_{2}^{\prime} \frac{\text { closed }}{\text { ext. }}>G_{2}$

By definition of $g(G, z)$ and $\mathcal{X}(G, \Sigma,, k)$ we get $G_{0} \in \mathcal{g}\left(G_{1}, v_{2}-\Sigma_{2}\right) \& G_{0} \in \mathcal{X}\left(G_{2}, \Sigma_{1},\left\|G_{1}\right\|\right)$. 
This shows, that the existence of such a diagram is equivalent to

$$
g\left(G_{1}, v_{2}-\Sigma_{2}\right)>\mathcal{L}\left(\sigma_{2}, \Sigma_{1},\left\|G_{1}\right\|\right) \neq \phi
$$

Using lemma 4 and lemma 5 we can decide this last relation. This completes the proof of our

Theorem 3: For any two contextfree grammars $G_{1}$ and $G_{2}$ it is decidable wether or not $G_{1} \equiv G_{2}$ holds.

Remark: We have shown, that the relation

$$
\lambda^{()}\left(G_{2}\right) E \lambda^{()}\left(G_{1}\right) \text { is aeciaable, too. }
$$




\section{REFERENCES:}

[1] E. Bertsch, An Observation on Relative Parsing Time, JACM (2) 4, 1975, 493-498

[2] A. Cremers - S. Ginsburg, Contextfree Grammar Forms JCSS $11,1975,86-117$

[3] s. Ginsburg, The Mathematical Theory of Contextfree Languages, McGraw-Hill, Book Co., New York, 1966

[4] G. Hotz, Eindeutigkeit und Mehrdeutigkeit formaler Sprachen, EIK(2) 4, 1966, 235-246

[5] R. MCNaughton, Parenthesis Grammars, JACM 14, 1967, 490-500

[6] A. Salomaa, Formal Languages, Academic Press, New York, 1971

[7] C.P. Schnorr, Vier Entscheidbarkeitsprobleme für kontextsensitive Sprachen, Computing 93, 1968, 311-317

[8] C.P. schnorr, On Transformational Classes of Grammars, Inf. and Control 14, 1969, 252-277

[9] H. Walter, Verallgemeinerte Pullbackkonstruktionen bei Semi-Thue-Systemen, EIK 6, (4/5), 1970 , 239-254

[10] H. Walter, Grammar Forms and Grammar Morphisms, to appear in Acta Informatica, 1976

[11] H. Walter, Gramatik-und sprachfamilien, Teil IV TB AFS 75-22, TH Dafmstad, 1975 